\title{
B1524-136: A CSS quasar with two-sided radio jets
}

\author{
F. Mantovani ${ }^{1}$, D. J. Saikia ${ }^{2}$, M. Bondi ${ }^{1}$, W. Junor ${ }^{3}$, C. J. Salter ${ }^{4}$, and R. Ricci ${ }^{5}$ \\ 1 Istituto di Radioastronomia, CNR, via P. Gobetti 101, Bologna, Italy \\ 2 National Centre for Radio Astrophysics, TIFR, Post Bag 3, Ganeshkhind, Pune 411 007, India \\ 3 Department of Physics and Astronomy, University of New Mexico, 800 Yale Blvd., N.E., Albuquerque, \\ NM 87131, USA \\ 4 Arecibo Observatory, HC3 Box 53995, Arecibo, Puerto Rico PR 00612, USA \\ 5 Scuola Internazionale Superiore di Studi Avanzati, via Beirut 4, 34014 Trieste, Italy
}

Received 10 April 2002 / Accepted 7 May 2002

\begin{abstract}
We present MERLIN, global VLBI and VLBA observations of the high-luminosity, compact steepspectrum quasar B1524-136 at cm wavelengths. These observations reveal well-defined radio jets on both sides of the active nucleus, a situation which is almost unique amongst high-luminosity radio quasars. However, the radio jets on opposite sides are very dissimilar, and the overall radio structure appears highly distorted. We discuss possible implications of these observations.
\end{abstract}

Key words. galaxies: active - galaxies: jets - galaxies: nuclei - quasars: general - quasars: individual: B1524-136 radio continuum: galaxies

\section{Introduction}

Although most high-luminosity, extragalactic radio sources belonging to Fanaroff-Riley class II (FRII) have two reasonably symmetric lobes of radio emission on opposite sides of the parent optical object, the radio jets in these sources are overwhelmingly one-sided (cf. Bridle \& Perley 1984). This is particularly true for quasars where radio jets are detected much more frequently than in radio galaxies. For example, in a study of 12 extended 3CR quasars with the Very Large Array (VLA), Bridle et al. (1994) made deep images with typical rms noises of $20 \mu \mathrm{Jy} /$ beam. They detected jets in all 12 sources and candidate counter-jets in 7 . However, there is no unambiguous counter-jet in any of these sources. In a similar study of a sample of 13 radio galaxies by Fernini et al. $(1993,1997)$, only two have radio jets and neither of these show evidence of any counter jet. Although radio jets are not frequently detected in FRII radio galaxies, two of the good examples of counter-jets seen in FRII radio sources are associated with relatively nearby radio galaxies. One of these is the well-studied classical double-lobed source, Cygnus A, which is at a redshift of 0.056. A jet towards the north-west from the nucleus and a faint counter-jet towards the south-east can be seen on both parsec and kiloparsec scales along a position angle, $\mathrm{PA}=284 \pm 2^{\circ}$ (Perley et al. 1984; Linfield 1985; Carilli et al. 1996;

Send offprint requests to: F. Mantovani,

e-mail: fmantova@ira.bo.cnr.it
Krichbaum et al. 1996; Sorathia et al. 1996). Another FRII radio galaxy where a well-collimated jet and counter-jet have been detected is $3 \mathrm{C} 353$, which is also at a relatively small redshift of 0.03 (Swain et al. 1998).

Radio jets are believed to be signatures of the beams carrying energy from the nucleus to the outer lobes, and there have been a number of suggestions to explain the apparent asymmetry of the jets despite the outer lobes being reasonably symmetric. These include the possibilities that (i) the jets are intrinsically symmetric but appear one-sided or highly asymmetric because the brightness of the receding one is diminished due to bulk relativistic motion while the approaching jet is boosted for the same reason (Rees 1978; Blandford \& Königl 1979; Scheuer \& Readhead 1979); (ii) the active beam on the counter-jet side has a low synchrotron emissivity and (iii) the energy supply is intrinsically asymmetric, but supplies energy alternately on opposite sides of the nucleus (cf. Saikia \& Wiita 1982; Rudnick \& Edgar 1984).

There appears to be a consensus of opinion that the observed asymmetry or one-sidedness of the jets is due to bulk relativistic motion. Although early arguments relied on the strong correlation of jet detection with core strength (cf. Saikia 1984) and the parsec- and kiloparsecscale jets being on the same side (cf. Bridle \& Perley 1984), the strongest evidence comes from the correlation of depolarization asymmetry with jet sidedness (Laing 1988; Garrington et al. 1988; Garrington et al. 1991). This would also be consistent with jets being seen more frequently in 
quasars and also being very asymmetric and one-sided in these, since quasars are believed to be inclined at smaller angles to the line of sight than radio galaxies (cf. Barthel 1989).

In this paper, using observations made with milliarcsec resolution, we report the detection of two-sided jets in a compact steep-spectrum (CSS) quasar. The CSSs are defined to be of subgalactic dimensions, with an overall size $\lesssim 20 \mathrm{kpc}$ and a steep high-frequency spectrum having $\alpha \geq 0.5$, where $S \propto \nu^{-\alpha}$. They are believed to be young sources at an early stage of evolution (Fanti et al. 1995; Readhead et al. 1996a,b; O'Dea 1998; Owsianik \& Conway 1998). We present below the results of our observations of the CSS quasar B1524-136 and discuss their implications.

\section{1. $B 1524-136$}

The radio source B1524-136 is associated with a quasar at a redshift of 1.687 , so that $1^{\prime \prime}$ corresponds to $8.44 \mathrm{kpc}$ in a Universe with $H_{\mathrm{o}}=50 \mathrm{~km} \mathrm{~s}^{-1} \mathrm{Mpc}^{-1}$ and $q_{\mathrm{o}}=0.5$. It has a steep radio spectrum $\left(\alpha_{750}^{5000} \approx 0.64\right)$ which appears to flatten below a few hundred $\mathrm{MHz}$ (cf. Steppe et al. 1995). It is a well-known low-frequency variable source (Bondi et al. 1996 and references therein), although it does not exhibit significant variations at $\mathrm{cm}$ wavelengths (Padrielli et al. 1987). VLA observations at $5 \mathrm{GHz}$ find B1524-136 to be slightly extended, while at $15 \mathrm{GHz}$ it is resolved into a double source (Mantovani et al. 1994). Interplanetary scintillation observations at $327 \mathrm{MHz}$ show that about 50 per cent of the total flux density is from a compact structure with a size of $\lesssim 100$ milliarcsec (Jeyakumar et al. 2000).

\section{Radio observations}

MERLIN The source was observed with MERLIN on 1995 May 06 at $5 \mathrm{GHz}$ for $\approx 8 \mathrm{hr}$ using a bandwidth of $28 \mathrm{MHz}$. Initial values for the telescope and correlator gains were determined from a short observation of the bright unresolved calibrator B0552+398 (DA193). The primary flux density and polarization calibrator was 3C286 and the secondary calibrator was B1511+100.

Global VLBI MK2 The global VLBI MK2 observations were made on 1992 September 21 with a central frequency of $1663.99 \mathrm{MHz}$ and a $2-\mathrm{MHz}$ bandwidth. The array consisted of antennas at Onsala, Effelsberg, Westerbork, Jodrell Bank (the Lovell telescope), Medicina, Green Bank, Haystack, VLBA-KP, VLBA-PT, VLBA-LA, VLBA-NL, VLBA-BR and VLBA$\mathrm{OV}$. The source was tracked for a total of $7.5 \mathrm{hr}$ in a snap-shot observing programme. The data were processed at the Caltech Block2 correlator.

VLBA These observations were carried out on 1996 Feb. 04 with the Very Long Baseline Array (VLBA) and one antenna of the VLA recording both right- and leftcircular polarization (RCP, LCP) using one-bit sampling. The source was observed at $8.4 \mathrm{GHz}$ for $4.3 \mathrm{hr}$ with a

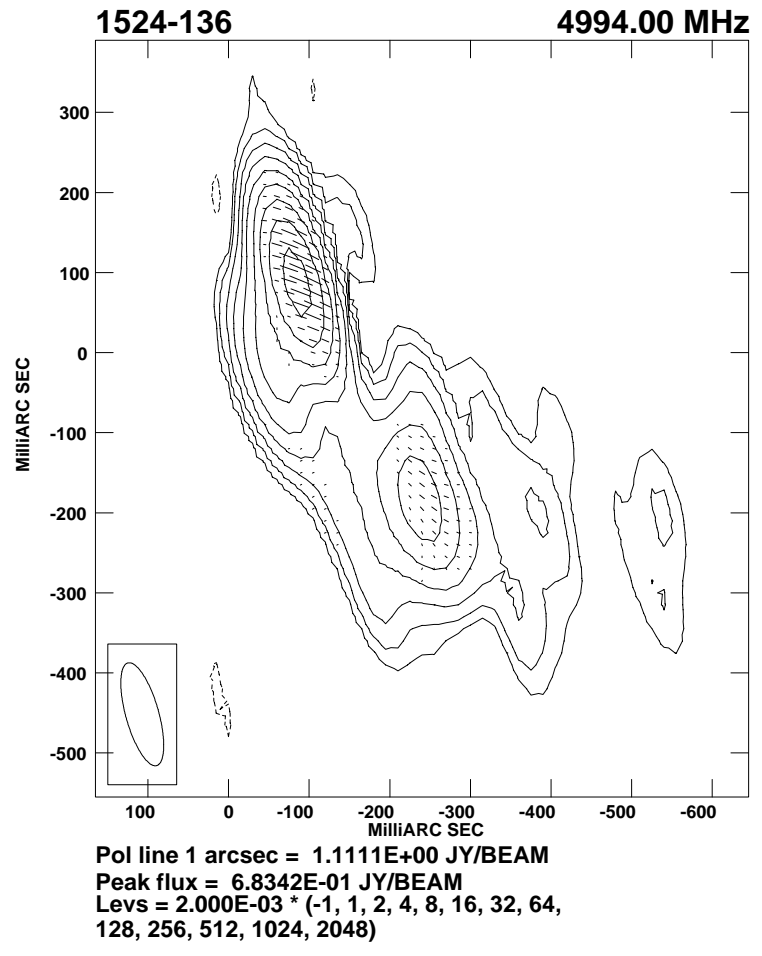

Fig. 1. The The MERLIN 5-GHz image with an angular resolution of $133 \times 41$ mas along a PA of $16^{\circ}$. The total-intensity contours are overlayed by vectors representing the linearly polarized intensity. The vector orientations give the polarization position angle.

bandwidth of $32 \mathrm{MHz}$. Amplitude calibration was derived from measurements of the system temperature during the observations and the known antenna gain of each element of the array. Complex correlation coefficients were recovered at the Array Operations Center in Socorro. Phase gradients in frequency and time were corrected by the use of global fringe fitting (Schwab \& Cotton 1983).

\section{The radio images}

Earlier VLA A-array observations of B1524-136 at $5 \mathrm{GHz}$ show the source to be slightly extended, while the $15-\mathrm{GHz}$ observations show it to be clearly double-lobed with an angular size of 0.32 arcsec along a PA of $30^{\circ}$. The weaker southern component is 4.3 per cent polarized at $15 \mathrm{GHz}$ compared to 2.5 per cent for the northern component (Mantovani et al. 1994). Recent polarization observations with the VLBA show the northern jet and hot-spot to have very high rotation measures (RMs) in the range of -3000 to $-10000 \mathrm{rad} \mathrm{m}^{-2}$ in the rest frame of the source (Mantovani et al. 2002).

The MERLIN 5-GHz image with an angular resolution of $133 \times 41$ mas along a PA of $16^{\circ}$ and an rms noise of $0.3 \mathrm{mJy} /$ beam is shown in Fig. 1. This image is of higher resolution than the VLA images and reveals greater details of the structure. The two main components, northern and southern, are clearly seen and are 4.4 and 7.9 per cent polarized respectively. The peak brightness of the northern component is higher than the southern one by a factor of 


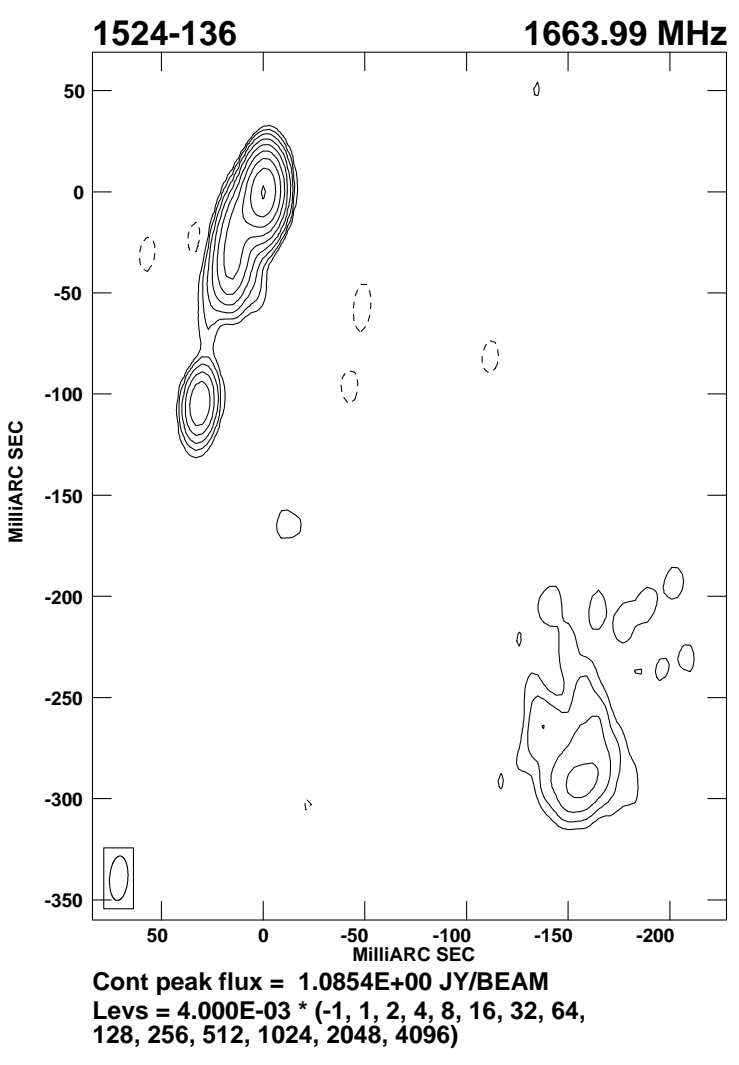

Fig. 2. The global MK2 VLBI image at $1.7 \mathrm{GHz}$ with an angular resolution of $22 \times 9$ mas along a PA of $-4^{\circ}$.

about 7 . In addition, there is a barely-resolved component south of the northern component which does not show signficant polarized emission, the degree of polarization being $\lesssim 1$ per cent. This suggests that this component is likely to be the nucleus of the active galaxy.

The global MK2 VLBI image at $1.7 \mathrm{GHz}$ (Fig. 2), with an angular resolution of $22 \times 9$ mas along a PA of $-4^{\circ}$ and an rms noise of $1.3 \mathrm{mJy} /$ beam, reveals the core clearly, making B1524-136 a highly non-collinear radio source. The supplement of the angle formed at the core by the outer hotspots is $62^{\circ}$. Such distorted structures are not uncommon among CSS objects (cf. Saikia et al. 1995, 2001) and are perhaps caused by deflection of one or both jets by dense clouds of gas. However, intrinsic distortions may appear amplified by projection effects since the object is associated with a quasar and may thus be inclined at a relatively small angle to the line of sight. The peak and total core flux density estimated by fitting a two-dimensional Gaussian are $128 \mathrm{mJy} /$ beam and $140 \mathrm{mJy}$ respectively. At this resolution, the northern hot-spot is brighter than the southern one by a factor of about 25 . In addition to the core and the hot-spots there is evidence of a jet which connects the core to the northern hotspot. By fitting twodimensional Gaussians to the hot-spot and jet, and taking slices across the jet, we estimate the width of the jet to be about 7 mas and its length to be about 35 mas. This qualifies it to be called a jet using the definition of Bridle \& Perley (1984). There is, however, a gap or region of

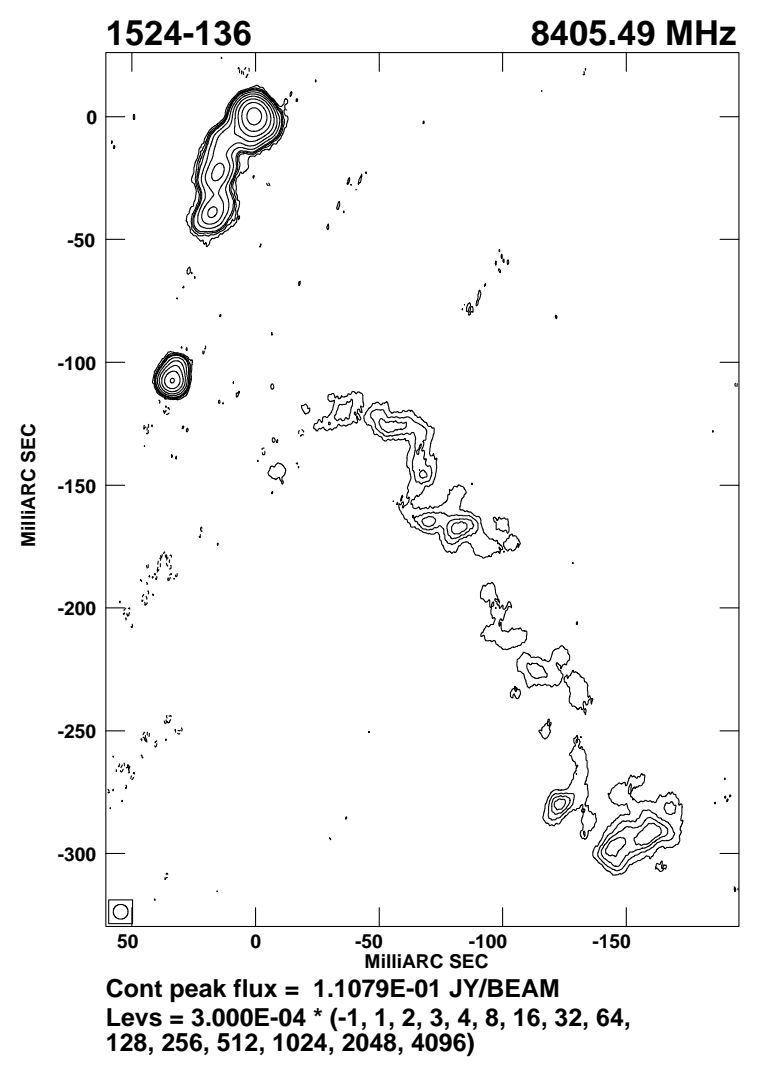

Fig. 3. The VLBA image at $8.4 \mathrm{GHz}$ restored with a circular beam of 6 mas.

very low emission between the core and the beginning of the radio jet. The hot-spot has a size of about 7 mas and an integrated flux density of $1400 \mathrm{mJy}$, and it must be the dominant component contributing to the scintillating flux density at $327 \mathrm{MHz}$ (Jeyakumar et al. 2000) and lowfrequency variability at $408 \mathrm{MHz}$ (Bondi et al. 1996).

The VLBA image at $8.4 \mathrm{GHz}$ has the highest resolution of our images, 6 mas, and is also the most sensitive one with an rms noise of $100 \mu \mathrm{Jy} /$ beam. It shows a couple of prominent knots in the jet, which appears to bend by about $40^{\circ}$ before entering the hot-spot (Fig. 3). The core is clearly extended to the north along the direction of the jet. A two-dimensional Gaussian fit shows the core to have peak and total flux densities of $40 \mathrm{mJy} /$ beam and $48 \mathrm{mJy}$ respectively, and the deconvolved size to be about 4 mas along a $\mathrm{PA}=165^{\circ}$. The core is unresolved in the orthogonal direction. The peak flux density of the core at $1.7 \mathrm{GHz}$ and its total flux density at $8.4 \mathrm{GHz}$ yields a spectral index of 0.6. However, the spectrum is likely to be flatter than this because of the coarser resolution at the lower frequency and the presence of a jet-like structure close to the nucleus. There is a gap of emission of about 50 mas between the nuclear jet and the beginning of the more extended one which connects to the dominant hot-spot.

The most striking feature of the VLBA image is the dectection of a well-defined counter-jet which exhibits some three cycles of oscillation before entering the southern hot-spot. Here too there appears to be a gap of about 
60 mas between the core and the beginning of the counterjet. This is perhaps the clearest detection yet of a jet and a counter-jet in a quasar. The mean brightness of the knots in the counter-jet is weaker than the main jet by a factor of about 11, while the peak brightness of the jet-side hotspot is brighter than the one on the counter-jet side by a factor of about 70 .

\section{Discussion}

The most striking result of these observations of B1524-136 is the detection of both a jet and a counter-jet in a quasar. Assuming that the unified scheme for powerful radio galaxies and quasars is broadly correct (cf. Barthel 1989), the object should be inclined at less than about $45^{\circ}$ to the line of sight. The fraction of emission from the radio core, $f_{\mathrm{c}}$, at an emitted frequency of $8 \mathrm{GHz}$ is 0.09 , assuming a spectral index of 0 for the core and 0.7 for the extended emission. The equivalent value of $R$, the ratio of the core to the extended flux density, is 0.10. Adopting the values of $R_{\mathrm{T}}=0.0008$ and $\gamma=10$, which give a reasonable fit to the observed distribution of $R$ in FRII 3CR sources (cf. Saikia \& Kulkarni 1994), the observed value of $R$ for B1524-136 implies an angle of inclination to the line of sight, $\theta \sim 20^{\circ}$. This value does not change significantly even if we assume the core spectral index to be 0.5 . Here $R_{\mathrm{T}}$ is the value of $R$ transverse to the line-of-sight and $\gamma$ is the bulk Lorentz factor. The ratio of the mean brightness of the knots in the jet to those in the counter-jet is 11 , which implies a bulk velocity for the jet of $\approx 0.5 \mathrm{c}$ if the apparent brightness asymmetry is entirely due to relativistic beaming. This velocity estimate increases by only about 15 per cent even if the counter-jet is bent away from the jet axis by as much as $40^{\circ}$.

The jetted side is closer to the nucleus, which is not consistent with expectations of a symmetric external environment (cf. Saikia 1981; Scheuer 1995). This hot-spot being closer and brighter is possibly due to a higher dissipation of energy on the jetted side due to interaction with a much denser external medium on the northern side. Although we do not have information to determine the RM of the southern hot-spot, evidence of such a dense medium on the northern side can be seen in the RM of this region which ranges from -3000 to $-10000 \mathrm{rad} \mathrm{m}^{-2}$ in the rest frame of the source (Mantovani et al. 2002). The brightness asymmetry of the hot-spots in the VLBA image is higher than that of the jets by a factor of about 6 . Even if the hot-spots are travelling close to the speed of the jets, the additional asymmetry in brightness would require the density in the northern side to be higher than the southern one by about 40 using a model for the propagation of jets in an asymmetric environment (Eilek \& Shore 1989; Gopal-Krishna \& Wiita 1991; Saikia et al. 1996).

The oscillations seen in the counter-jet are reminescent of the development of Kelvin-Helmholtz instabilities (cf. Hardee 1987). For a helical oscillation $(n=1)$, we can estimate the Mach number of the jet using the relation for the fastest growing oscillation or resonant wavelength given by $\lambda_{h} / r \sim 5.2 M /\left(0.66+\eta^{1 / 2}\right)$ where $\lambda_{h}$ is the wavelength of the helical oscillations, $r$ is the jet radius, $M$ is the Mach number and $\eta$ is the ratio of the jet density to the density of the external medium. Estimating the average jet radius to be 3.5 mas by fitting two-dimensional Gaussians to the brighter knots in the counter-jet, we find the Mach number to be 2.5 for a light jet $(\eta \rightarrow 0)$ and 6.3 in the highly entrained limit $(\eta \rightarrow 1)$.

The gaps in emission between the core and both the jet and counter-jet could be due to quasi-continuous activity in the nucleus. The gap of about 50 mas, a jet speed of $0.5 \mathrm{c}$ and an angle of inclination of $20^{\circ}$ indicates a time scale of $\approx 10^{4} \mathrm{yr}$ for this quasi-continuous activity. This discontinuity is on a much smaller time scale than the repeated encounters with external galaxies or mergers which may completely re-ignite radio activity in the nucleus leading to the formation of double-double radio sources. The typical time scale for these events is several Myr (cf. Schoenmakers et al. 2000, and references therein).

Although we have considered just a simple scenario above to explain the large-scale properties of the source, the situation could be far more complicated. The large projected misalignment between the two jets, plus the oscillations in the counter-jet, suggest that the bending is three-dimensional, and that different sections of both jets could be inclined at different angles to the line of sight. Also, the jets could decelerate as they traverse outwards from the nucleus, and the degree of deceleration could be different on opposite sides of the nucleus. These would all affect the observed brightness ratio of the jets.

Considering the main jet, its PA is aligned within a few degrees of the extension seen in the core and it is reasonable to assume that the jet is straight till it bends by about $40^{\circ}$ before entering the hotspot. For a jet traversing outwards with a velocity $v=\beta c$, corresponding to a Lorentz factor, $\gamma$, the observed flux density of even the approaching jet will be Doppler diminished when $\gamma(1-\beta \cos \theta)>1$. As noted earlier, the $3 \mathrm{CRR}$ radio sources suggest a bulk Lorentz factor, $\gamma \sim 10$. The observed flux density of the approaching jet would then be Doppler diminished for an angle of inclination $\theta \gtrsim 25^{\circ}$; the corresponding brightness ratio being $>2000$. A deceleration of the jets to about $0.5 \mathrm{c}$ by the region where the jets are first detected would result in a decrease in Doppler dimming and might provide a viable explanation of the existence of gaps and the detection of the radio jets at about 50 mas from the nucleus.

We can test this scenario further by examining whether it provides a satisfactory explanation to the ratios of the brightnesses of the outer jets to about 3 times the rms noise in the gaps of emission. The observed ratio is $\gtrsim 35$ for the approaching jet, and $\gtrsim 3$ for the counter-jet. Assuming the jets to be intrinsically of reasonably uniform brightness along their lengths, the velocities discussed earlier would require the angle of inclination to be about $45^{\circ}$, which is the dividing line between radio galaxies and quasars in the unified scheme (Barthel 1989). However, although this is consistent with the unified scheme, 
the observed value of core prominence would imply that the intrinsic value, $\mathrm{R}_{T}$ would have to be about 20 times greater than the canonical value of 0.0008 mentioned above. It is therefore conceivable that the intrinsic brightness of the jet is lower in the gaps, and this could be further diminished by the effects of relativistic beaming.

A plausible scenario is one in which the jet is inclined at about $25^{\circ}$ to the line of sight, and the counter-jet is bent by about $50^{\circ}$ such that it is about $75^{\circ}$ to the line of sight. This will decrease the degree of Doppler dimming, making the counter-jet visible, and will also help explain the large observed misalignment of the source. The velocity required to explain the brightness asymmetry of the jets is then $\approx 0.6 \mathrm{c}$. Although this does not exhaust the range of scenarios that might explain the situation, it provides a reasonable explanation of the large-scale features of the source. A deeper image to detect radio emission in the gaps between the nucleus and the jets, and proper motion studies of the knots should enable us to build a better model for the source.

\section{Concluding remarks}

We report the detection of a well-defined radio jet and a counter-jet in the compact steep-spectrum quasar B1524-136, which is almost unique in a radio quasar. We present MERLIN, global VLBI and VLBA observations of the source. The radio source is highly non-collinear and asymmetric, and was reported recently to have a high RM of several thousand $\mathrm{rad} \mathrm{m}^{-2}$ on the northern side. An angle of inclination of about $25^{\circ}$, a jet speed of about $0.6 \mathrm{c}$, an intrinsic misalignment of about $50^{\circ}$, such that the counterjet is at about $75^{\circ}$ to the line of sight, and a density asymmetry on opposite sides of the nucleus by a factor of $\approx 40$ provides a consistent explanation of the observed largescale properties of the source.

Acknowledgements. We thank an anonymous referee for his valuable suggestions to widen the scope of the discussions. The Very Long Baseline Array and the Very Large Array are facilities of the National Radio Astronomy Observatory, USA, operated by Associated Universities, Inc., for the National Science Foundation. MERLIN is a UK national facility operated by the University of Manchester on behalf of PPARC. We thank the staff of the different observatories which made these observations possible. We have made use of the NASA/IPAC Extragalactic Database (NED), which is operated by the Jet Propulsion Laboratory, California Institute of Technology under contract with the National Aeronautics and Space Administration.

\section{References}

Barthel, P. D. 1989, ApJ, 336, 606

Blandford, R. D., \& Königl, A. 1979, ApJ, 232, 34

Bondi, M., Padrielli, L., Fanti, R., et al. 1996, A\&AS, 120, 89

Bridle, A. H., \& Perley, R. A. 1984, ARA\&A, 22, 319

Bridle, A. H., Hough, D. H., Lonsdale, C. J., Burns, J. O., \& Laing, R. A. 1994, AJ, 108, 766
Carilli, C. L., Perley, R. A., Bartel, N., \& Dreher, J. W. 1996, Cygnus A - A study of a radio galaxy, ed. C. L. Carilli, \& D. E. Harris (Cambridge University Press), 76

Eilek, J. A., \& Shore, S. N. 1989, ApJ, 342, 187

Fanti, C., Fanti, R., Dallacasa, D., et al. 1995, A\&A, 302, 317 Fernini, I., Burns, J. O., Bridle, A. H., \& Perley, R. A. 1993, AJ, 105, 1690

Fernini, I., Burns, J. O., \& Perley, R. A. 1997, AJ, 114, 2292

Garrington, S. T., Conway, R. G., \& Leahy, J. P. 1991, MNRAS, 250, 171

Garrington, S. T., Leahy, J. P., Conway, R. G., \& Laing, R. A. 1988, Nature, 331, 147

Gopal-krishna, \& Wiita, P. J. 1991, ApJ, 373, 325

Hardee, P. E. 1987, ApJ, 313, 607

Jeyakumar, S., Saikia, D. J., Rao, A. P., \& Balasubramanian, V. 2000, A\&A, 362, 27

Krichbaum, T. P., Alef, W., \& Witzel, A. 1996, Cygnus A A study of a radio galaxy, ed. C. L. Carilli, \& D. E. Harris (Cambridge University Press), 92

Laing, R. A. 1988, Nature, 331, 149

Linfield, R. 1985, ApJ, 295, 463

Mantovani, F., Junor, W., Fanti, R., Padrielli, L., \& Saikia, D. J. 1994, A\&A, 292, 59

Mantovani, F., Junor, W., Ricci, R., et al. 2002, A\&A, 389, 58

O'Dea, C. P. 1998, PASP, 110, 493

Owsianik, I., \& Conway, J. E. 1998, A\&A, 337, 69

Padrielli, L., Aller, M. F., Aller, H. D., et al. 1987, A\&AS, 67, 63

Perley, R. A., Dreher, J. W., \& Cowan, J. J. 1984, ApJ, 285, L35

Readhead, A. C. S., Taylor, G. B., Xu, W., et al. 1996a, ApJ, 460,612

Readhead, A. C. S., Taylor, G. B., Pearson, T. J., \& Wilkinson, P. N. 1996b, ApJ, 460, 634

Rees, M. J. 1978, Nature, 275, 516

Rudnick, L., \& Edgar, B. K. 1984, ApJ, 279, 74

Saikia, D. J. 1981, MNRAS, 197, 11P

Saikia, D. J. 1984, MNRAS, 208, 231

Saikia, D. J., \& Kulkarni, V. K. 1994, MNRAS, 270, 897

Saikia, D. J., \& Wiita, P. J. 1982, MNRAS, 200, 83

Saikia, D. J., Jeyakumar, S., Wiita, P. J., Sanghera, H. S., \& Spencer, R. E. 1995, MNRAS, 276, 1215

Saikia, D. J., Jeyakumar, S., Wiita, P. J., \& Hooda, J. S. 1996. The second workshop on Gigahertz peaked spectrum and Compact steep spectrum radio sources, ed. I. A. G. Snellen, R. T. Schilizzi, H. J. A. Röttgering, \& M. N. Bremer, (Sterrewacht Leiden), 252

Saikia, D. J., Jeyakumar, S., Salter, C. J., et al. 2001, MNRAS, 321,37

Scheuer, P. A. G. 1995, MNRAS, 277, 331

Scheuer, P. A. G., \& Readhead, A. C. S. 1979, Nature, 277, 182

Schoenmakers, A. P., de Bruyn, A. G., Röttgering, H. J. A., van der Laan, H., \& Kaiser, C. R. 2000, MNRAS, 315, 371

Schwab, F. R., Cotton, W. D. 1983, AJ, 88, 688

Sorathia, B., Bartel, N., Bietenholtz, M. F., \& Carilli, C. L. 1996, Cygnus A - A study of a radio galaxy, ed. C. L. Carilli, \& D. E. Harris (Cambridge University Press), 86

Steppe, H., Jeyakumar, S., Saikia, D. J., \& Salter, C. J. 1995, A\&AS, 113, 409

Swain, M. R., Bridle, A. H., \& Baum, S. A. 1998, ApJ, 507, $29 \mathrm{~L}$ 